Supporting Information for

\title{
Targeted Synthesis of Uranium(IV) Thiosilicates
}

Vladislav V. Klepov, Mark D. Smith, Hans-Conrad zur Loye*

Department of Chemistry and Biochemistry, University of South Carolina, Columbia, South Carolina, 29208, United States 


\section{Experimental section}

Caution: Although the uranium precursor used in this synthesis contains depleted uranium, it is required that proper procedures for handling radioactive materials are observed. All handling of radioactive materials was performed in labs specially designated for the study of radioactive actinide materials.

Synthesis. U turnings (International Bio-Analytical Industries, ACS grade), S powder (Fisher Scientific, sublimed), Si (99.5\%, Alfa Aesar), $\mathrm{Na}_{2} \mathrm{~S}$ (Alfa Aesar), CsI (99.9\%, Alfa Aesar), and NaI (99.9\%, Alfa Aesar) were all used as received.

Uranium sulfide $\mathrm{US}_{2}$ was obtained by a direct reaction between uranium metal turnings and elemental sulfur powder in a stoichiometric ratio. The turnings were rinsed with concentrated nitric acid to wash away uranium oxides from their surface. Then the turnings were rinsed several times with acetone and dried in a vacuum desiccator. A mixture of $1.00 \mathrm{~g}$ of $\mathrm{U}$ and $0.2694 \mathrm{~g}$ of S (1:2 molar ratio) was loaded into a carbon-coated silica tube in an argon glovebox. The tube with a $10 \mathrm{~mm}$ inner diameter was flame-sealed under a pressure of $<10^{-4}$ Torr and placed vertically in a programmable box furnace at room temperature. The approximate length of the sealed tube is $12 \mathrm{~cm}$. The furnace was ramped up to $650{ }^{\circ} \mathrm{C}$ at a rate of $5{ }^{\circ} \mathrm{C} / \mathrm{h}$ and kept at this temperature for 48 hours. The slow heating rate is necessary to avoid pressure buildup inside the tube from sulfur volatilization that will readily burst the fused silica tube by letting the sulfur react completely with the uranium foil. After the reaction was completed, the tube was cooled to room temperature by switching off the furnace. The powder X-ray diffraction pattern of the resulting black powder revealed no impurities.

Silicon sulfide was obtained in a similar fashion by a direct reaction between Si and stoichiometric amount of S. Si (1.00 g) and S (2.28 g) powders were thoroughly ground together and loaded into a silica tube, which was sealed under a pressure of $<10^{-4}$ Torr. The tube was loaded in a tube furnace which was ramped up to $600^{\circ} \mathrm{C}$ at a rate of $0.2^{\circ} \mathrm{C} / \mathrm{min}$ and dwelled at this temperature for $30 \mathrm{~h}$. The resulting hard grey product was evacuated from the tube and stored in a nitrogen-filled glovebag. The PXRD pattern of the product identifies poorly crystallized $\mathrm{SiS}_{2}$ as a single phase.

The starting reagents, $\mathrm{US}_{2}(0.100 \mathrm{~g}), \mathrm{Na}_{2} \mathrm{~S}(0.052 \mathrm{~g}), \mathrm{SiS}_{2}(0.122 \mathrm{~g})$ in a 1:2:4 molar ratio and $1.00 \mathrm{~g}$ of eutectic CsI-NaI and CsI fluxes for $\mathrm{Cs}_{2} \mathrm{Na}_{4}\left[\mathrm{U}_{2}\left(\mathrm{SiS}_{4}\right)_{2}\left(\mathrm{Si}_{2} \mathrm{~S}_{8}\right)\right]$ and to $\mathrm{Cs}_{2.12} \mathrm{Na}_{3.88}\left[\mathrm{U}_{2}\left(\mathrm{SiS}_{4}\right)\left(\mathrm{Si}_{2} \mathrm{~S}_{7}\right)\right]$, respectively, were mixed inside a carbon-coated silica tube in a nitrogen glovebag. The reagent filled silica tubes were flame sealed under a vacuum of $<10^{-4}$ 
Torr and placed vertically into a programmable box furnace. The furnace was ramped up to 600 and $750^{\circ} \mathrm{C}$, for CsI-NaI and CsI fluxes, respectively, in $1.5 \mathrm{~h}$ and held at this temperature for 20 h. Following this heating step, the furnace was cooled to below the melting point of the flux at a rate of $10^{\circ} \mathrm{C} / \mathrm{h}$ to effect the formation of crystallized products. Once at room temperature, the tube was cut open and the end of the tube containing the reaction products was placed into a beaker filled with DMF. After soaking the reaction products for several hours the crystals were isolated by filtration. A sample of $\mathrm{Cs}_{2} \mathrm{Na}_{4}\left[\mathrm{U}_{2}\left(\mathrm{SiS}_{4}\right)_{2}\left(\mathrm{Si}_{2} \mathrm{~S}_{8}\right)\right]$, which was obtained from CsI-NaI flux, was slightly $(<5 \%)$ contaminated with $\mathrm{US}_{3}$ as indicated by additional peaks in the PXRD pattern (Figure S1). CsI flux at $750^{\circ} \mathrm{C}$ gave rise to $\mathrm{Cs}_{2.12} \mathrm{Na}_{3.88}\left[\mathrm{U}_{2}\left(\mathrm{SiS}_{4}\right)\left(\mathrm{Si}_{2} \mathrm{~S}_{7}\right)\right]$ single crystals along with a significant amount of UOS impurity.

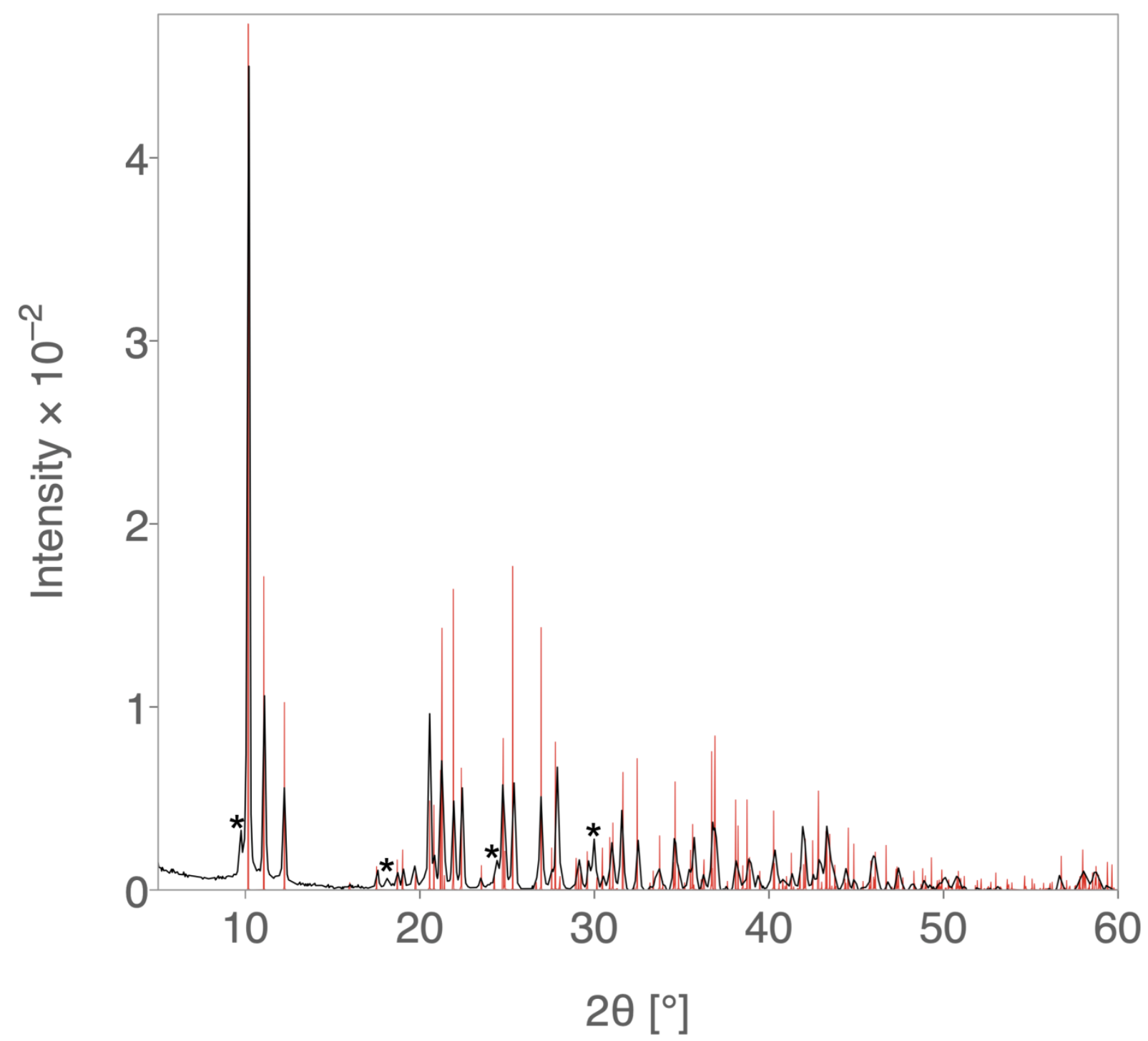

Figure S1. Experimental (black) and calculated (red) PXRD pattern of $\mathrm{Cs}_{2} \mathrm{Na}_{4}\left[\mathrm{U}_{2}\left(\mathrm{SiS}_{4}\right)_{2}\left(\mathrm{Si}_{2} \mathrm{~S}_{8}\right)\right]$. $\mathrm{US}_{3}$ impurity peaks marked with asterisk. 


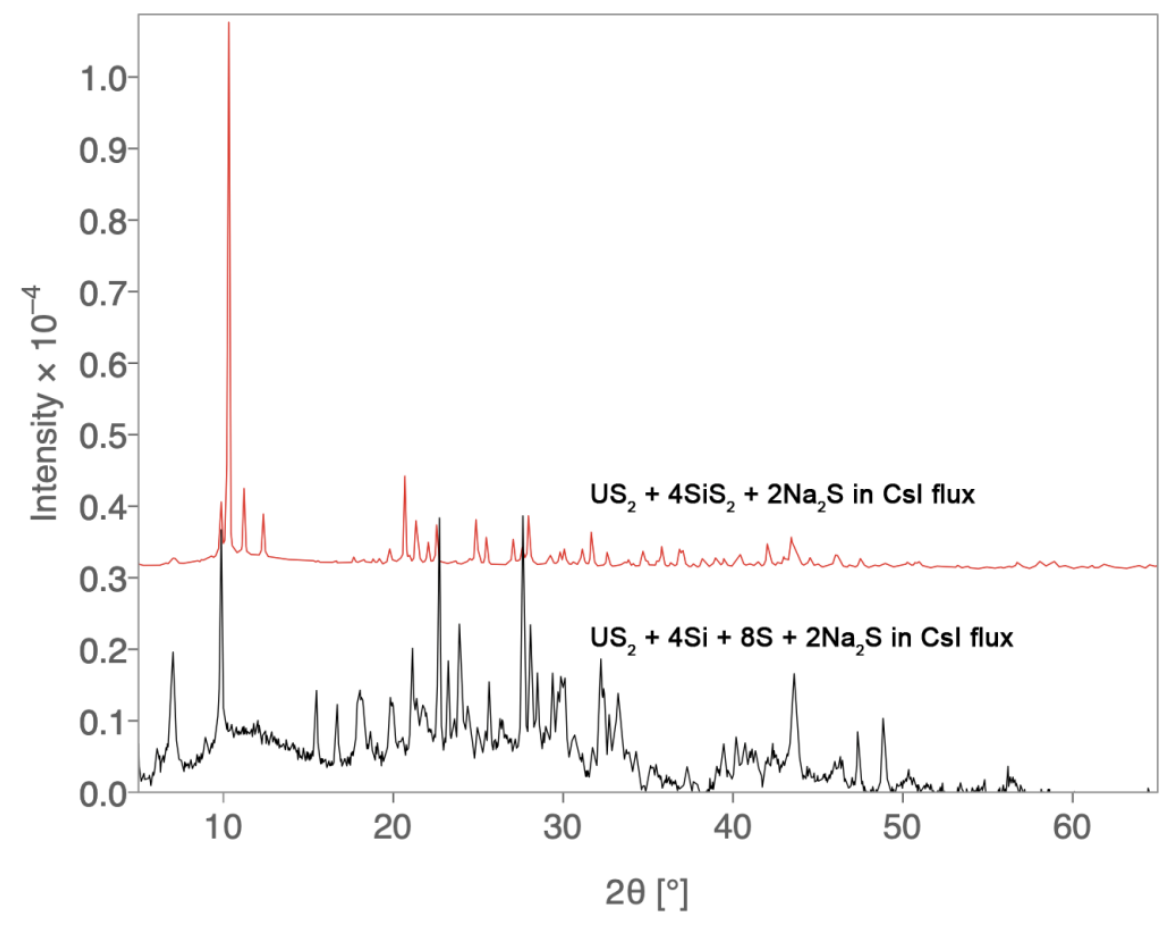

Figure S2. PXRD patterns of the products obtained from reactions employing $\mathrm{SiS}_{2}$ (top) or a respective stoichiometric amount of $\mathrm{Si}$ and $\mathrm{S}$ (bottom). $\mathrm{Cs}_{2} \mathrm{Na}_{4}\left[\mathrm{U}_{2}\left(\mathrm{SiS}_{4}\right)_{2}\left(\mathrm{Si}_{2} \mathrm{~S}_{8}\right)\right]$ was observed only in the case when $\mathrm{SiS}_{2}$ was used as a silicon source.

Single crystal X-ray crystallography. Single-crystal X-ray diffraction data were collected at 100(2) K on a Bruker D8 QUEST diffractometer equipped with an Incoatec I $\mu$ S 3.0 microfocus radiation source (MoK $\alpha, \lambda=0.71073 \AA$ ) and a PHOTON II area detector. The crystals were mounted on a microloop with immersion oil. The raw data reduction and absorption correction were performed using SAINT and SADABS programs. ${ }^{1,2}$ Full-matrix least-square refinements against $\mathrm{F}^{2}$ were performed with SHELXL software. ${ }^{3}$ All the structures were checked for missing symmetry with the Addsym program implemented into PLATON software and no higher symmetry was found. ${ }^{4}$ The crystallographic characteristics and results of the diffraction experiments are summarized in Table S1.

In both structures, pyrothiosilicate groups were found to be disordered. $\mathrm{S}_{2} \mathrm{~S}_{8}{ }^{6-}$ anion in $\mathbf{1}$ is disordered over two positions by an inversion center, while in $\mathbf{2}, \mathrm{S}_{2} \mathrm{~S}_{7}{ }^{6-}$ anions have severely disordered bridging sulfur atom, and two positions for each of the silicon atoms. The terminal sulfur atoms thermal ellipsoids are elongated accordingly to the silicon atoms disorder, further supporting the proposed disorder model.

The disorder in these crystals raised our attention to possible twinning. We carefully checked the single crystal diffraction data for possible non-merohedral twinning, but almost 
perfect indexation and ideal reflection shape precluded this option. One could surmise merohedral twinning in the crystal, i.e. with all the reflections from a twinned components perfectly overlapping with the reflections of a major phase. For $\mathrm{Cs}_{2} \mathrm{Na}_{4} \mathrm{U}_{2}\left(\mathrm{SiS}_{4}\right)_{2} \mathrm{Si}_{2} \mathrm{~S}_{8}$, we tried to solve the structure in lower symmetry space groups $\mathrm{Cm}$ and $\mathrm{C} 2$ and find a twinning law, but the disorder persisted, which led us to a conclusion that the disorder is intrinsic to these crystals.

Table S1. Atomic positions, Wyckoff symbols, and site occupancies of the atoms in $\mathrm{Cs}_{2} \mathrm{Na}_{4}\left[\mathrm{U}_{2}\left(\mathrm{SiS}_{4}\right)_{2}\left(\mathrm{Si}_{2} \mathrm{~S}_{8}\right)\right]$

\begin{tabular}{|l|c|c|c|c|c|c|}
\hline Atom & $\begin{array}{c}\text { Wyckoff } \\
\text { position, site- } \\
\text { symmetry }\end{array}$ & $\mathrm{x}$ & $\mathrm{y}$ & $\mathrm{z}$ & $\mathrm{U}_{\text {iso }}$ & Occupancy \\
\hline $\mathrm{U} 1$ & $4 \mathrm{~h}, \mathrm{C}_{2}$ & 0.5 & $0.24661(2)$ & 0.5 & $0.00939(3)$ & 1 \\
\hline $\mathrm{Cs} 1$ & $4 \mathrm{i}, \mathrm{C}_{\mathrm{s}}$ & $0.76461(2)$ & 0.5 & $0.57350(2)$ & $0.01684(4)$ & 1 \\
\hline $\mathrm{S} 1$ & $4 \mathrm{i}, \mathrm{C}_{\mathrm{s}}$ & $0.51790(4)$ & 0 & $0.33129(8)$ & $0.01069(10)$ & 1 \\
\hline $\mathrm{S} 2$ & $4 \mathrm{i}, \mathrm{C}_{\mathrm{s}}$ & $0.34954(5)$ & 0 & $-0.01781(8)$ & $0.01413(12)$ & 1 \\
\hline $\mathrm{S} 3$ & $8 \mathrm{j}, \mathrm{C}_{1}$ & $0.33850(3)$ & $0.17929(5)$ & $0.30654(5)$ & $0.01114(8)$ & 1 \\
\hline S4 & $4 \mathrm{i}, \mathrm{C}_{\mathrm{s}}$ & $0.58082(5)$ & 0.5 & $0.64891(9)$ & $0.01605(12)$ & 1 \\
\hline $\mathrm{S} 5$ & $8 \mathrm{j}, \mathrm{C}_{1}$ & $0.42744(12)$ & $0.3202(3)$ & $0.7274(2)$ & $0.0140(3)$ & 0.5 \\
\hline S5B & $8 \mathrm{j}, \mathrm{C}_{1}$ & $0.40614(12)$ & $0.3153(3)$ & $0.6932(2)$ & $0.0140(3)$ & 0.5 \\
\hline S6 & $4 \mathrm{i}, \mathrm{C}_{\mathrm{s}}$ & $0.58881(10)$ & 0.5 & $0.89003(17)$ & $0.0169(2)$ & 0.5 \\
\hline S7 & $4 \mathrm{i}, \mathrm{C}_{\mathrm{s}}$ & $0.59940(10)$ & 0.5 & $1.00144(17)$ & $0.0195(3)$ & 0.5 \\
\hline Si1 & $4 \mathrm{i}, \mathrm{C}_{\mathrm{s}}$ & $0.38222(5)$ & 0 & $0.22427(8)$ & $0.00964(12)$ & 1 \\
\hline Si2 & $4 \mathrm{i}, \mathrm{C}_{\mathrm{s}}$ & $0.50072(10)$ & 0.5 & $0.77412(18)$ & $0.0130(3)$ & 0.5 \\
\hline Si3 & $4 \mathrm{i}, \mathrm{C}_{\mathrm{s}}$ & $0.45149(11)$ & 0.5 & $0.80906(18)$ & $0.0133(3)$ & 0.5 \\
\hline $\mathrm{Na} 1$ & $4 \mathrm{~g}, \mathrm{C}_{2}$ & 0.5 & $0.17089(15)$ & 1 & $0.0259(3)$ & 1 \\
\hline $\mathrm{Na2}$ & $4 \mathrm{e}, \mathrm{C}_{\mathrm{i}}$ & 0.25 & 0.25 & 0 & $0.024(3)$ & $0.24(7)$ \\
\hline $\mathrm{Na3}$ & $4 \mathrm{e}, \mathrm{C}_{\mathrm{i}}$ & $0.2305(14)$ & $0.2238(19)$ & $-0.0024(5)$ & $0.024(3)$ & $0.38(4)$ \\
\hline
\end{tabular}

Table S2. Atomic positions, Wyckoff symbols, and site occupancies of the atoms in $\mathrm{Cs}_{2.12} \mathrm{Na}_{3.88}\left[\mathrm{U}_{2}\left(\mathrm{SiS}_{4}\right)\left(\mathrm{Si}_{2} \mathrm{~S}_{7}\right)\right]$

\begin{tabular}{|c|c|c|c|c|c|c|}
\hline Atom & $\begin{array}{c}\text { Wyckoff position, } \\
\text { site-symmetry }\end{array}$ & $\mathrm{x}$ & $\mathrm{y}$ & $\mathrm{z}$ & $\mathrm{U}_{\text {iso }}$ & Occupancy \\
\hline U1 & $8 \mathrm{f}, \mathrm{C}_{1}$ & $0.37383(2)$ & $0.62437(2)$ & $0.49618(2)$ & $0.01742(4)$ & 1 \\
\hline Cs1 & $8 \mathrm{f}, \mathrm{C}_{1}$ & $0.36937(2)$ & $0.12400(3)$ & $0.46004(2)$ & $0.02125(6)$ & 1 \\
\hline Cs2 & 4e, $\mathrm{C}_{2}$ & 0.5 & $0.0036(3)$ & 0.25 & $0.0241(6)$ & 0.12 \\
\hline S1 & $8 f, \mathrm{C}_{1}$ & $0.26177(5)$ & $0.46544(10)$ & $0.40453(6)$ & $0.01951(18)$ & 1 \\
\hline S2 & $8 f, \mathrm{C}_{1}$ & $0.08136(5)$ & $0.64686(10)$ & $0.41092(6)$ & $0.02128(18)$ & 1 \\
\hline S3 & 8f, $\mathrm{C}_{1}$ & $0.63510(6)$ & $0.59807(11)$ & $0.39711(7)$ & $0.0272(2)$ & 1 \\
\hline S4 & 8f, $\mathrm{C}_{1}$ & $0.25776(6)$ & $0.81762(10)$ & $0.41210(6)$ & $0.02118(19)$ & 1 \\
\hline S5 & $8 f, \mathrm{C}_{1}$ & $0.44457(7)$ & $0.75500(12)$ & $0.37360(7)$ & $0.0325(3)$ & 1 \\
\hline S6 & $8 f, \mathrm{C}_{1}$ & $0.46425(6)$ & $0.41657(11)$ & $0.42119(9)$ & $0.0361(3)$ & 1 \\
\hline S7 & $8 f, \mathrm{C}_{1}$ & $0.1833(2)$ & $0.6698(3)$ & $0.24122(18)$ & $0.0253(6)$ & 0.5 \\
\hline S8 & $8 f, \mathrm{C}_{1}$ & $0.16749(17)$ & $0.6212(3)$ & $0.24091(17)$ & $0.0188(5)$ & 0.5 \\
\hline S9 & 4e, $\mathrm{C}_{2}$ & $0.5103(14)$ & $0.4735(5)$ & $0.2489(15)$ & $0.045(3)$ & $0.344(3)$ \\
\hline S10 & 4e, $\mathrm{C}_{2}$ & $0.5072(18)$ & $0.5630(16)$ & $0.2605(10)$ & $0.045(3)$ & $0.088(2)$ \\
\hline S10B & $8 f, \mathrm{C}_{1}$ & $0.5501(18)$ & $0.502(3)$ & $0.2538(15)$ & $0.045(3)$ & $0.067(3)$ \\
\hline
\end{tabular}




\begin{tabular}{|c|c|c|c|c|c|c|}
\hline $\mathrm{Si} 1$ & $8 \mathrm{f}, \mathrm{C}_{1}$ & $0.19224(6)$ & $0.64076(12)$ & $0.36403(6)$ & $0.0215(2)$ & 1 \\
\hline $\mathrm{Si} 2 \mathrm{~A}$ & $8 \mathrm{f}, \mathrm{C}_{1}$ & $0.5124(9)$ & $0.5888(15)$ & $0.3474(7)$ & $0.0164(10)$ & $0.36(3)$ \\
\hline $\mathrm{Si} 2 \mathrm{~B}$ & $8 \mathrm{f}, \mathrm{C}_{1}$ & $0.5123(4)$ & $0.5748(9)$ & $0.3638(7)$ & $0.0164(10)$ & $0.64(3)$ \\
\hline $\mathrm{Na} 1$ & $8 \mathrm{f}, \mathrm{C}_{1}$ & $0.2904(4)$ & $0.4212(5)$ & $0.2464(2)$ & $0.0462(11)$ & 0.5 \\
\hline $\mathrm{Na} 2$ & $8 \mathrm{f}, \mathrm{C}_{1}$ & $0.3382(2)$ & $0.8055(4)$ & $0.2358(2)$ & $0.0327(8)$ & 0.5 \\
\hline $\mathrm{Na} 3$ & $8 \mathrm{f}, \mathrm{C}_{1}$ & $0.3278(2)$ & $0.4837(5)$ & $0.2476(2)$ & $0.0315(8)$ & 0.5 \\
\hline $\mathrm{Na} 4$ & 4e, $\mathrm{C}_{2}$ & 0.5 & $0.1866(4)$ & 0.25 & $0.0403(8)$ & 0.88 \\
\hline
\end{tabular}

Table S3. Crystallographic data for $\mathrm{Cs}_{2} \mathrm{Na}_{4}\left[\mathrm{U}_{2}\left(\mathrm{SiS}_{4}\right)_{2}\left(\mathrm{Si}_{2} \mathrm{~S}_{8}\right)\right](\mathbf{1})$ and $\mathrm{Cs}_{2.12} \mathrm{Na}_{3.88}\left[\mathrm{U}_{2}\left(\mathrm{SiS}_{4}\right)\left(\mathrm{Si}_{2} \mathrm{~S}_{7}\right)\right]$ (2).

\begin{tabular}{|c|c|c|}
\hline Chemical formula & $\mathrm{Cs}_{2} \mathrm{Na}_{4}\left[\mathrm{U}_{2}\left(\mathrm{SiS}_{4}\right)_{2}\left(\mathrm{Si}_{2} \mathrm{~S}_{8}\right)\right]$ & $\mathrm{Cs}_{2.12} \mathrm{Na}_{3.88}\left[\mathrm{U}_{2}\left(\mathrm{SiS}_{4}\right)_{2}\left(\mathrm{Si}_{2} \mathrm{~S}_{7}\right)\right]$ \\
\hline Formula weight & 1459.16 & 1440.29 \\
\hline Crystal system & Monoclinic & Monoclinic \\
\hline Space group, $\mathrm{Z}$ & $C 2 / m$ & $C 2 / c$ \\
\hline $\mathrm{a}, \AA$ & $16.6640(6)$ & $16.7459(5)$ \\
\hline $\mathrm{b}, \AA$ & $9.7115(3)$ & $9.6776(3)$ \\
\hline $\mathrm{c}, \AA$ & $9.1164(3)$ & $17.0008(5)$ \\
\hline$\beta$, deg. & $109.9330(10)$ & $97.5250(10)$ \\
\hline $\mathrm{V}, \AA^{3}$ & $1386.94(8)$ & $2731.42(14)$ \\
\hline$\rho_{\text {calcd, }} \mathrm{g} / \mathrm{cm}^{3}$ & 3.49 & 3.50 \\
\hline Radiation $(\lambda, \AA)$ & \multicolumn{2}{|c|}{ Mo K $\alpha 0.71073$} \\
\hline$\mu, \mathrm{mm}^{-1}$ & 15.686 & 16.007 \\
\hline $\mathrm{T}, \mathrm{K}$ & $100(2)$ & $100(2)$ \\
\hline Crystal dim., $\mathrm{mm}^{3}$ & $0.04 \times 0.06 \times 0.14$ & $0.06 \times 0.12 \times 0.14$ \\
\hline $2 \theta$ range, deg. & $2.468-33.171$ & $2.647-29.999$ \\
\hline Reflections collected & 35708 & 54001 \\
\hline Data/parameters/restraints & $2776 / 97 / 0$ & $3983 / 169 / 1$ \\
\hline$R_{\text {int }}$ & 0.0131 & 0.0141 \\
\hline Goodness of fit & 1.116 & 1.083 \\
\hline $\mathrm{R}_{1}(\mathrm{I}>2 \sigma(\mathrm{I}))$ & 0.0141 & 0.0208 \\
\hline $\mathrm{wR}_{2}$ (all data) & 0.0354 & 0.0409 \\
\hline
\end{tabular}




\section{Powder X-ray Diffraction}

Powder X-ray diffraction (PXRD) data for phase identification and phase purity confirmation were collected on polycrystalline samples. Data were collected on a Bruker D2 PHASER diffractometer utilizing $\mathrm{Cu} \mathrm{K} \alpha$ radiation. The data were collected over the range from $10^{\circ}$ to $65^{\circ} 2 \theta$ with a step size of $0.02^{\circ}$. The PXRD pattern is shown on Figure S1. Several impurity peaks correspond to $\mathrm{US}_{3}$.

\section{Energy-Dispersive Spectroscopy (EDS)}

EDS was performed on product single crystals using a Tescan Vega-3 SEM equipped with a Thermo EDS attachment (Figure S2). The SEM was operated in low-vacuum mode. Crystals were mounted on an SEM stub with carbon tape and analyzed using a $20 \mathrm{kV}$ accelerating voltage and an $80 \mathrm{~s}$ accumulating time. The results of EDS confirm the presence of elements found by single-crystal X-ray diffraction (Table S2).
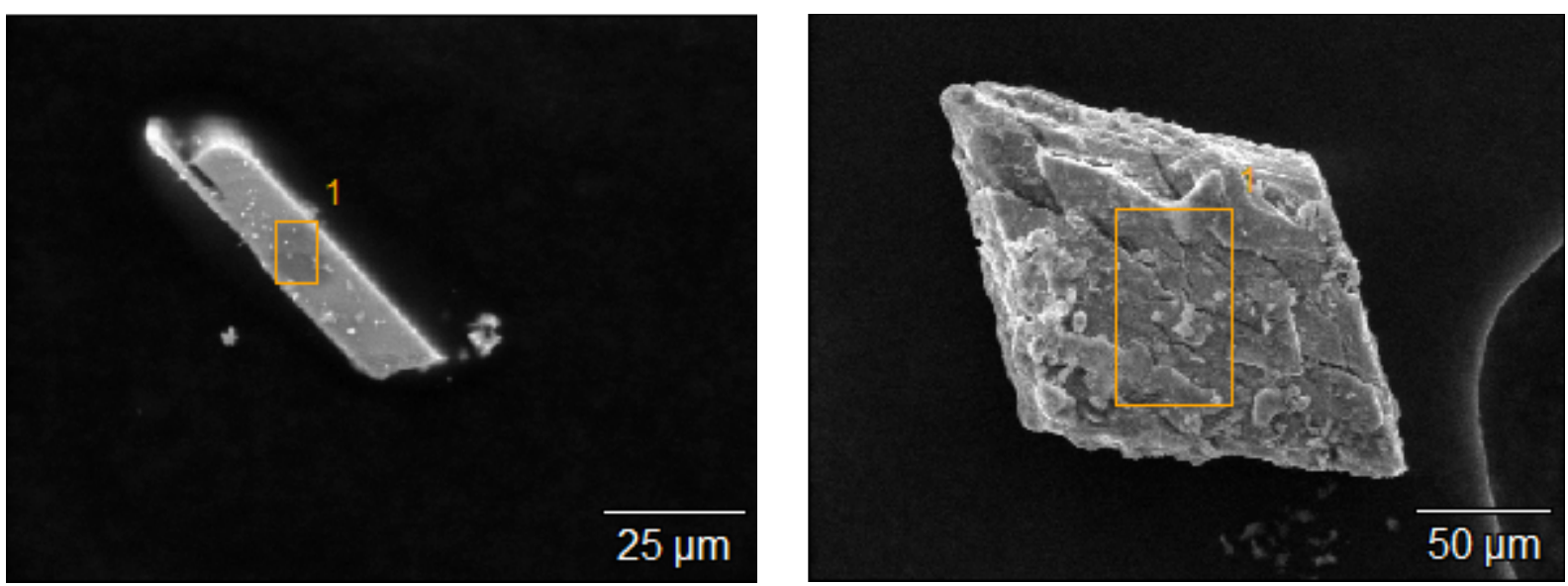

Figure S3. SEM images of $\mathrm{Cs}_{2} \mathrm{Na}_{4}\left[\mathrm{U}_{2}\left(\mathrm{SiS}_{4}\right)_{2}\left(\mathrm{Si}_{2} \mathrm{~S}_{8}\right)\right](\mathrm{left})$ and $\mathrm{Cs}_{2.12} \mathrm{Na}_{3.88}\left[\mathrm{U}_{2}\left(\mathrm{SiS}_{4}\right)\left(\mathrm{Si}_{2} \mathrm{~S}_{7}\right)\right]$ (right).

Table S4. Elemental compositions of $\mathrm{Cs}_{2} \mathrm{Na}_{4}\left[\mathrm{U}_{2}\left(\mathrm{SiS}_{4}\right)_{2}\left(\mathrm{Si}_{2} \mathrm{~S}_{8}\right)\right]$ and $\mathrm{Cs}_{2.12} \mathrm{Na}_{3.88}\left[\mathrm{U}_{2}\left(\mathrm{SiS}_{4}\right)\left(\mathrm{Si}_{2} \mathrm{~S}_{7}\right)\right]$ as determined by EDS.

\begin{tabular}{|c|c|c|c|}
\hline \multicolumn{2}{|c|}{$\mathrm{Cs}_{2} \mathrm{Na}_{4}\left[\mathrm{U}_{2}\left(\mathrm{SiS}_{4}\right)_{2}\left(\mathrm{Si}_{2} \mathrm{~S}_{8}\right)\right]$} & \multicolumn{2}{|c|}{$\mathrm{Cs}_{2.12} \mathrm{Na}_{3.88}\left[\mathrm{U}_{2}\left(\mathrm{SiS}_{4}\right)\left(\mathrm{Si}_{2} \mathrm{~S}_{7}\right)\right]$} \\
\hline Element & Atom \% & Element & Atom \% \\
\hline $\mathrm{Na}$ & 19.55 & $\mathrm{Na}$ & 27.52 \\
\hline $\mathrm{Si}$ & 14.48 & $\mathrm{Si}$ & 21.38 \\
\hline $\mathrm{S}$ & 53.08 & $\mathrm{~S}$ & 26.04 \\
\hline
\end{tabular}




\begin{tabular}{|c|c|c|c|}
\hline $\mathrm{Cs}$ & 6.44 & $\mathrm{Cs}$ & 18.06 \\
\hline $\mathrm{U}$ & 6.46 & $\mathrm{U}$ & 7.00 \\
\hline
\end{tabular}

\section{Magnetism}

Magnetic property measurements were performed on a Quantum Design MPMS 3 SQUID magnetometer. Zero-field-cooled magnetic susceptibility measurements were performed from 2 to $300 \mathrm{~K}$ in an applied field of $0.1 \mathrm{~T}$. The raw data were corrected for radial offset and sample shape effects according to the method described in the literature. ${ }^{5}$ The magnetic data were collected on polycrystalline powder obtained by grinding the product single crystals.

\section{Optical Properties}

UV-vis spectra were recorded using a Perkin Elmer Lambda 35 UV/visible scanning spectrophotometer used in the diffuse reflectance mode and equipped with an integrating sphere (Figures S3). Diffuse reflectance spectra were recorded in the 200-900 nm range. Reflectance data were converted to absorbance using the Kubelka-Munk function.

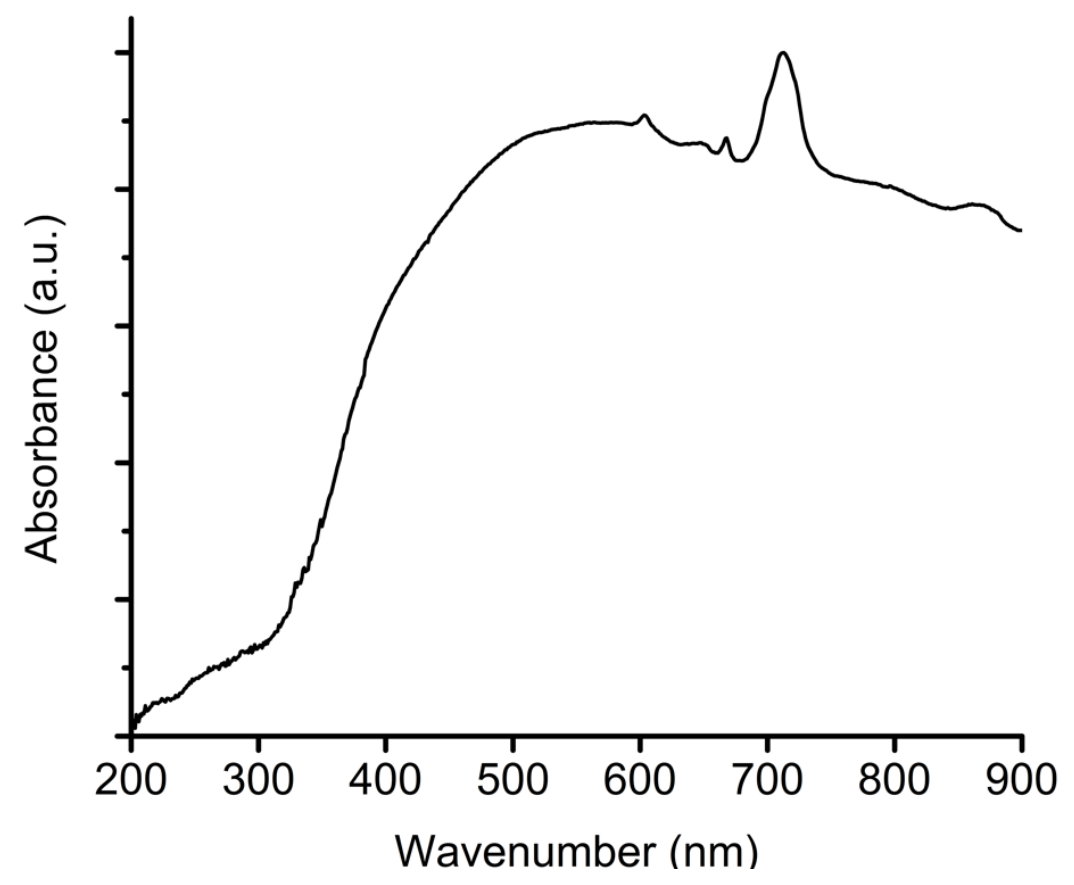

Figure S4. UV-vis spectrum of $\mathrm{Cs}_{2} \mathrm{Na}_{4}\left[\mathrm{U}_{2}\left(\mathrm{SiS}_{4}\right)_{2}\left(\mathrm{Si}_{2} \mathrm{~S}_{8}\right)\right]$. 